\title{
Analysis of Hedging Profits under Two Stock Pricing Models
}

\author{
Lingyan Cao ${ }^{1}$, Zheng-Feng Guo ${ }^{2}$ \\ ${ }^{1}$ Department of Mathematics, University of Maryland, Maryland, USA \\ ${ }^{2}$ Department of Economics, Vanderbilt University, Nashville, USA \\ E-mail: \{lingyancao,gzfsadie\}@gmail.com \\ Received August 29, 2011; revised October 13, 2011; accepted October 22, 2011
}

\begin{abstract}
In this paper, we employ two stock pricing models: a Black-Scholes (BS) model and a Variance Gamma (VG) model, and apply the maximum likelihood method (MLE) to estimate corresponding parameters in each model. With the estimated parameters, we conduct Monte Carlo simulations to simulate spot prices and deltas of the European call option at different time spots over different sample paths. We focus on calculateing the deltas for the two models by the method of the infinitesimal perturbation analysis (IPA). Then, we employ dynamic delta hedging using the simulated spot prices and deltas for different hedging periods. Finally, hedging profits under these two models are calculated and analyzed.
\end{abstract}

Keywords: Likelihood Ratio, Variance Gamma, Geometric Brownian Motion, Delta Hedge

\section{Introduction}

Delta hedging is a particular type of hedging strategy based on the Greek called Delta, which measures the sensitivity of option prices with respect to spot prices. A general introduction of delta hedging was provided in [1]; a detailed explanation of dynamic delta hedging and the process of how to replicate portfolios with delta hedging and achieve a delta-neutral position are studied in [2].

In order to employ delta hedging, we need to first estimate deltas. Generally, there are two types of techniques: direct and indirect methods. The former includes the infinitesimal perturbation analysis method (IPA) and the likelihood ratio method (LR). The latter includes finite difference methods, such as forward difference method, central difference method and backward difference method. However, the indirect methods require additional simulations and are sensitive to the choice of scalars. Thus, the direct methods are more popular in estimating Greeks. There is a vast literature in discussing the direct methods. The IPA was first applied to option pricing for both European and American options in [3,4] reviews various Monte Carlo methods for financial engineering; see also [5] reviews various methods of gradient estimation in stochastic simulation, including both direct and indirect methods, as well as [6]. The LR method was first applied to option pricing in [7] for European and Asian option. However, we only focus on estimating deltas by IPA method in this paper.

In this paper, we assume two stock pricing models, one is the classical Black-Scholes (BS) model, in which the stock prices follows a geometric Brownian motion (GBM) process; another is the Variance Gamma (VG) model, in which stock prices follow a VG model. The BS model was first introduced by [8] and [9] to the finance community as a model for stock prices and option pricing; while the VG process was introduced for log-price returns and option pricing by [10], and further developed in [11,12]. A general review of the VG process in the context of stochastic Monte Carlo simulation is shown in [13]. The estimations and comparisons of Greeks under different models by different methods are studied in [14-16]. The comparisons of profits from delta hedging under different models and through different methods are studied in $[17,18]$.

In this paper, we focus on developing gradient estimates, i.e. deltas, for a European call option of which stock prices follow a GBM process and a VG process, respectively. We apply the MLE to the historical data of some assets to estimate corresponding parameters, price European call options, and calculate the deltas according to different stochastic processes through IPA. The remainder of the paper is organized as follows. Section 2 provides an introduction of geometric Brownian motion and VG process and shows how to price stock prices under these two models. Section 3 reviews several methods of gradient 
estimation. Section 4 explains the delta hedging strategy and shows how to employ dynamic delta hedging to obtain a risk-neutral position. Section 5 provides an example of delta hedging, of which data are from real market.

\section{Geometric Brownian Motion and Variance Gamma}

\subsection{Geometric Brownian Motion Process}

A stochastic process $S_{t}$ follows a geometric Brownian motion if $\log \left(S_{t}\right)$ is a Brownian motion with initial value $\log \left(S_{0}\right)$. In the BS model, the price of the underlying stock $S_{t}$ following a geometric Brownian motion satisfies

$$
\frac{\mathrm{d} S_{t}}{S_{t}}=\mu \mathrm{d} t+\sigma \mathrm{d} W_{t}
$$

where $W_{t}$ is a standard Brownian motion. With dividend yield $q$, spot $S_{0}$, volatility $\sigma$ and drift $\mu=r-q$, we can obtain the stock price as:

$$
S_{t}=S_{0} \exp \left(\left(r-q-\sigma^{2}\right) t+\sigma W_{t}\right)
$$

The stock price $S_{t}$ can be simulated through

$$
S_{t}=S_{0} \exp \left(\left(r-q-\sigma^{2}\right) t+\sigma \sqrt{t} \tilde{\mathrm{Z}}\right)
$$

where $\tilde{Z}$ is a random variable following a standard normal distribution.

\subsection{Variance Gamma Process}

The VG process is a Levy process of independent and stationary increments. Let $\gamma_{t}^{(\mu, v)}$ be a Gamma process with drift parameter $\mu$ and variance parameter $v$. There are two ways to define the VG process:

1) The VG process is defined as a Gamma-timechanged Brownian motion subordinated by a Gamma process. The representation of VG process (say GVG) is:

$$
X_{t}=B_{\gamma_{t}^{(1, v)}}^{(\theta, \sigma)}=\theta \gamma_{t}^{(1, v)}+\sigma W_{\gamma_{t}^{(1, v)}}
$$

where $B_{t}=\mu t+\sigma W_{t}$ represents a brownian motion with constant drift rate $\mu$ and volatility $\sigma$.

2) The VG process is expressed as a difference of two Gamma processes. The representation of the VG process as a difference of two gamma processes (say DVG) is

$$
X_{t}=\gamma_{t}^{\left(\mu_{+}, v_{+}\right)}-\gamma_{t}^{\left(\mu_{-}, v_{-}\right)}
$$

where $\mu_{ \pm}=\left(\sqrt{\theta^{2}+2 \sigma^{2} / v} \pm \theta\right) / 2$, and $v_{ \pm}=\mu_{ \pm}^{2} v$.

Under the risk-neutral measure, with no dividends and a constant risk-free interest rate $r$, the stock price is given by

$$
S_{t}=S_{0} \exp \left((r+\omega) t+X_{t}\right)
$$

where $\omega=\ln \left(1-\theta v-\sigma^{2} v / 2\right) / v$.

\section{Infinitesimal Perturbation Analysis}

Simulation and gradient estimation have been useful in financial engineering applications. To employ delta hedging, we first need to estimate deltas, one of gradient estimates of the price of a European call option. Delta is one of the Greeks, which are quantities representing the sensitivities of derivatives, such as options. Each Greek letter measures a different dimension to the risk in an option position and the aim of a trader is to manage the Greeks so that all risks are acceptable. More precisely, delta $\Delta$ is defined as the rate of change of option prices with respect to the underlying asset price. Therefore, deltas can be calculated by taking the derivative of the option prices with respect to the spot price.

Assuming the objective function $V(\xi)$ depends on the parameter $\xi$, we focus on calculating:

$$
\frac{\mathrm{d} V(\xi)}{\mathrm{d} \xi}
$$

Suppose the objective function is an expectation of the sample performance measure $L$, that is:

$$
V(\xi)=E[L(X ; \xi)]
$$

where $X=X_{1}, X_{2}, \cdots, X_{n}$ are dependent on $\xi$, and $n$ is the fixed number of random variables. The expectation can be written as:

$$
E[L(X)]=\int L(X) \mathrm{d} F_{X}(X),
$$

where $F_{X}$ is the distribution of the input random variables $X$. If we use inverse transform method to simulate the input random variable $X$, we can re-write Equation (6) as

$$
E[L(X)]=\int_{0}^{1} L(X(\xi ; u)) \mathrm{d} u,
$$

where the parameter $\xi$ dependence is in the distribution $F_{X}$. IPA method originally comes from taking the derivative of Equation (7). Moreover, IPA estimates require the integrability condition which is easily satisfied when the performance function is continuous with respect to the given parameter. Assume we can interchange the expectation and differentiation. The IPA estimate is:

$$
\frac{\mathrm{d} E[L(X)]}{\mathrm{d} \xi}=\int_{0}^{1} \frac{\mathrm{d} L}{\mathrm{~d} X} \frac{\mathrm{d} X(\xi)}{\mathrm{d} \xi} \mathrm{d} u,
$$

and the IPA estimator is:

$$
\frac{\mathrm{d} L}{\mathrm{~d} X} \frac{\mathrm{d} X(\xi)}{\mathrm{d} \xi}
$$

From Lebesgue dominated convergence theorem, the condition of uniform integrability of $\frac{\mathrm{d} L}{\mathrm{~d} X} \frac{\mathrm{d} X(\xi)}{\mathrm{d} \xi}$ must 
be satisfied to make the interchangeability. We only apply IPA to calculate the gradient estimate in the following paper.

\section{Delta Hedging Strategy}

Delta hedging is a trading strategy which attains to maintain a delta-neutral portfolio dynamically by offsetting the delta of the option position through the delta of the stock position. Delta measures the sensitivity of the option price $f$ with respect to the stock price $S$. In other words, when the stock price changes by small amoun $\Delta S$, the option price would change by the discounted amount of $\Delta \times \Delta S$. The investor could hedge the risk by adjusting (long or short) the shares of stocks to achieve a delta-neutral portfolio position. However, as delta changes, the investor's risk-neutral position will change accordingly. Thus, we have to adjust the hedging position periodically, which is called rebalancing. If we could rebalance immediately when the stock price changes, perfect hedge is achieved. However, perfect hedge is always difficult to achieve. What we can do is to employ a dynamic hedging and make the hedging period as short as possible. In the following, we show the process of delta hedging by setting the portfolio's delta zero. Details of the procedure to conduct delta hedging can refer to [4,5].

\section{An Example of Delta Hedging}

European call option gives the buyer the right, not the obligation to buy certain amount of financial instrument from the seller at the maturity time for a certain strike price. Let $S_{t}$ be the stock price at $t, T$ be the maturity time, $K$ be the strike price, and $r$ be the risk-free interest rate. The price (value) of the European call option at $t$ is

$$
V_{T}=e^{-r T}\left(S_{T}-K\right)^{+}
$$

where $S_{T}$ can follow a GBM process or a VG process.

\subsection{Estimating under GBM}

Assuming the stock price $S_{t}$ follows a GBM process, we can simulate $S_{t}$ in the following way:

$$
S_{t}=S_{0} \exp \left(\left(r-q-\sigma^{2}\right) t+\sigma \sqrt{t} \tilde{Z}\right)
$$

where $Z$ represents the standard normal random variable. The estimator of delta for the European call option through IPA is

$$
\frac{\mathrm{d} V_{T}}{\mathrm{~d} S_{0}}=e^{-r T} 1_{\left\{S_{T} \geq K\right\}} \frac{\mathrm{d} S_{T}}{\mathrm{~d} S_{0}},
$$

where

$$
\frac{\mathrm{d} S_{T}}{v S_{0}}=\frac{S_{T}}{S_{0}}=\exp \left(\left(r-q-\sigma^{2}\right) t+\sigma \sqrt{t} \tilde{Z}\right) \text {. }
$$

\subsection{Estimating under VG}

Assuming the stock price $S_{t}$ follows a VG process, we can write $S_{t}$ as:

$$
S_{t}=S_{0} \exp \left((r+\omega) t+X_{t}\right)
$$

where $X_{t}$ follows a VG process. We have two different ways to represent the VG process $X_{t}$ as in Equations (2) and (3). Thus we estimate the deltas in the corresponding two ways. The estimators for deltas of European call option through IPA are:

- the estimate of delta (under GVG):

$$
\frac{\mathrm{d} V_{T}}{v S_{0}}=e^{-r T} 1_{\left\{S_{T} \geq K\right\}} \frac{\mathrm{d} S_{T}}{\mathrm{~d} S_{0}},
$$

where

$$
\frac{\mathrm{d} S_{T}}{\mathrm{~d} S_{0}}=\frac{S_{T}}{S_{0}}=\exp \left((r+\omega) T+X_{T}\right) .
$$

- the estimate of delta (under DVG):

$$
\frac{\mathrm{d} V_{T}}{\mathrm{~d} S_{0}}=e^{-r T} 1_{\left\{S_{T} \geq K\right\}} \frac{\mathrm{d} S_{T}}{\mathrm{~d} S_{0}},
$$

where

$$
\frac{\mathrm{d} S_{T}}{\mathrm{~d} S_{0}}=\frac{S_{T}}{S_{0}}=\exp \left((r+\omega) T+X_{T}\right) .
$$

\subsection{Numerical Experiment}

In this paper, we use the historical data in WRDS of the stock price of Apple Ltd. from Mar.10, 2008 to Sept.10, 2008. Assuming stock price follows a GBM and VG process respectively, we apply the MLE to estimate the corresponding parameters. Assuming the maturity time for the option is 38 days, i.e., $\tilde{T}=38 / 365$, the risk free interest rate minus the dividend rate be $r-q=0.02451$, we get the variance parameter $\sigma=0.38204$ for GBM; and get $\sigma=0.35873, v=0.00000262, \theta=-0.01011$ for VG process. The spot price is $\tilde{S}_{0}=151.61$ at $t=t_{0}$ and the strike price is $K=155$.

We simulate all the spot prices $\hat{S i}$ at all different spot times and the deltas on all hedging time spots. The algorithms to estimate spot prices and deltas in one sample path refer to [4]. We then conduct the simulation on 10,000 sample paths. Moreover, after having the corresponding spot prices and deltas on each sample path, we employ delta hedging technique to calculate net gains on each sample path.

Table 1 shows the summary statistics for the net profits by delta hedging only once initially, i.e., $\Delta t=\tilde{T}=38 / 365$ by the methods above. Table 2 shows the summary statistics for the net profits by delta hedging 7 days, i.e., $\Delta t=7 / 365$ in all the methods above is shown. Table 3 shows the summary statistics for the net profits by delta hedging every 3 days i.e., $\Delta t=3 / 365$ in all the methods 
Table 1. Summary statistics of net profits by delta hedging once.

\begin{tabular}{lccc}
\hline summary stat & GBM IPA & GVG IPA & DVG IPA \\
\hline mean & 3659.8 & 3944.2 & 4116.7 \\
std & 5353.3 & 5580.7 & 4530.9 \\
min & -21004 & -25285 & -31987 \\
max & 7562.9 & 7962.3 & 7148.1 \\
median & 7058.7 & 7575.3 & 6686.3 \\
kurtosis & 4.1588 & 4.4942 & 4.5388 \\
skewness & -1.3766 & v1.4405 & -1.9523 \\
\hline
\end{tabular}

Table 2. Summary statistics of net profits by delta hedging every 7 days.

\begin{tabular}{lccc}
\hline summary stat & GBM IPA & GVG IPA & DVG IPA \\
\hline mean & 4457.2 & 4763.0 & 4592.4 \\
std & 2558.5 & 2985.4 & 2857.1 \\
min & -8498 & -8183 & -8074 \\
max & 7330.7 & 9420.3 & 9527.4 \\
median & 5051.4 & 4857.9 & 4644.6 \\
kurtosis & 3.7302 & 2.5653 & 4.0837 \\
skewness & -1.0192 & -0.3517 & -0.5850 \\
\hline
\end{tabular}

Table 3. Summary statistics of net profits by delta hedging every 3 days.

\begin{tabular}{lccc}
\hline summary stat & GBM IPA & GVG IPA & DVG IPA \\
\hline mean & 4875 & 4993.4 & 4992.6 \\
std & 1594 & 2476 & 2227 \\
min & -3420 & -3590 & -4025 \\
max & 6828 & 9157 & 9901 \\
median & 5253 & 4854 & 4205 \\
kurtosis & 3.9906 & 2.1933 & 2.2340 \\
skewness & -1.0707 & -0.0631 & -0.05913 \\
\hline
\end{tabular}

above. Figures 1-3 show the graph of empirical cumulative distribution functions of the net profits through all the methods above.

\section{Conclusions}

By comparing the numerical results we obtained so far, we can make the following conclusions:

- The mean value of net gain from delta hedging every 7 days is larger than hedging only once in the initial time.

- The mean value of net gain from delta hedging every 3 days is larger than hedging every 7 days.

- The mean value of net gain from VG is larger than the mean from GBM.

- The mean value of net gain from GVG and DVG are close.

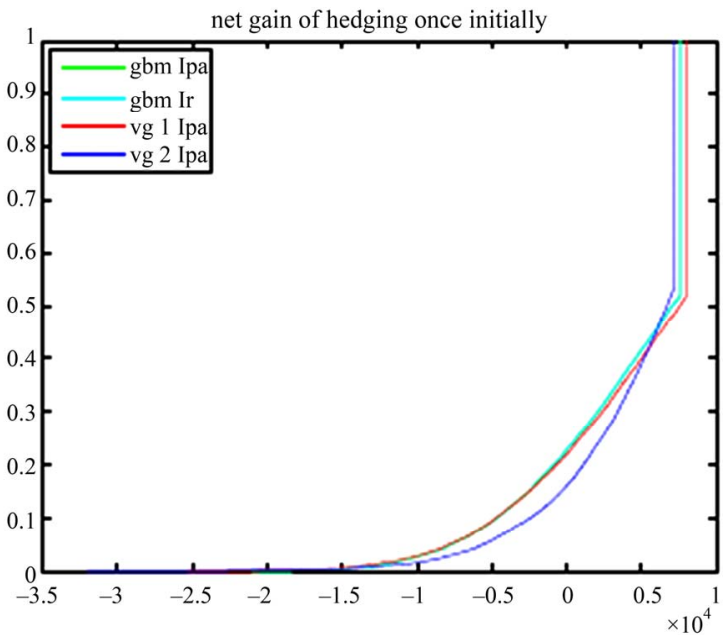

Figure 1. Empirical cdf of net profits of delta hedging once initially.

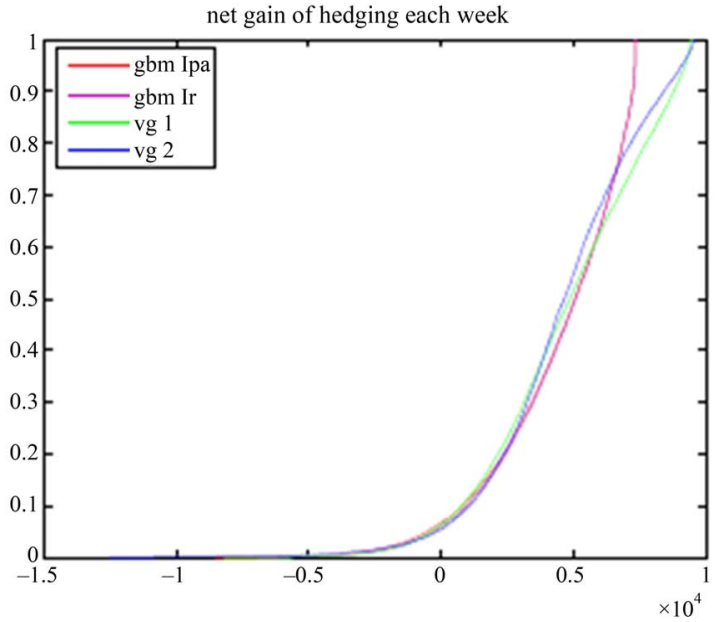

Figure 2. Empirical cdf of net profit of delta hedging every 7 days.

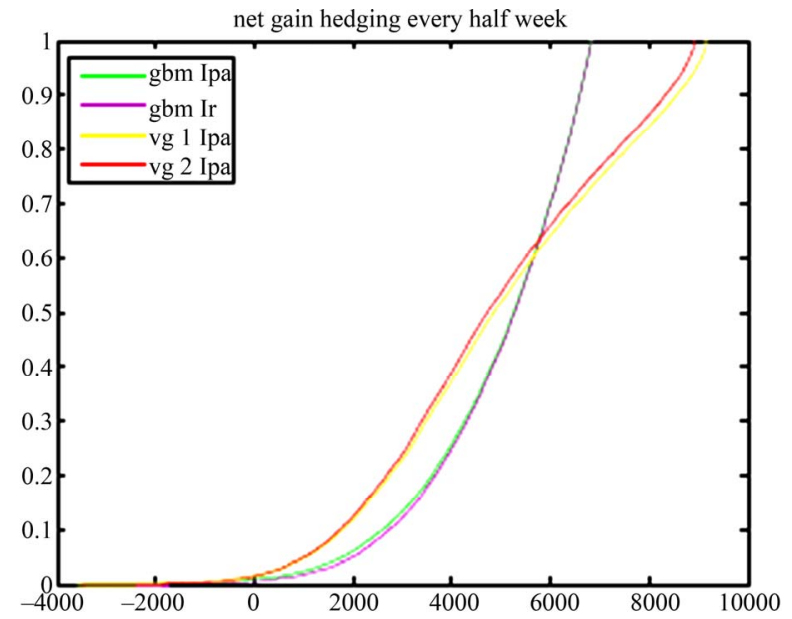

Figure 3. Empirical cdf of net profit of delta hedging every 3 days. 


\section{References}

[1] J. Hull, "Options, Futures, and Other Derivatives," 5th Edition, Prentice Hall, Upper Saddle River, 2003.

[2] R. Jarrow and S. Turnbull, "Derivative Securities," Thomson Learning Company, Belmont, 1999.

[3] M. C. Fu and J. Q. Hu, "Sensitivity Analysis for Monte Carlo Simulation of Option Pricing," Engineering and Informational Sciences, Vol. 9, No. 3, 1995, pp. 417-446. doi:10.1017/S0269964800003958

[4] P. Glasserman, "Monte Carlo Methods in Financial Engineering,” Springer, New York, 2004.

[5] M. C. Fu, "What You Should Know about Simulation and Derivatives,” Naval Research Logistics, Vol. 55, No. 8, 2006, pp. 723-736. doi:10.1002/nav.20313

[6] M. C. Fu, "Stochastic Gradient Estimation," In: S. G. Henderson and B. L. Nelson, Eds., Handbooks in Operations Research and Management Science, Elsevier, Amsterdam, 2008, pp. 575-616.

[7] M. Broadie and P. Glasserman, "Estimating Security Price Derivatives Using Simulation,” Management Science, Vol. 42, No. 2, 1996, pp. 269-285. doi:10.1287/mnsc.42.2.269

[8] F. Black and M. Scholes, "The Pricing of Options and Corporate Liabilities,” Journal of Political Economy, Vol. 81, No. 3, 1973, pp. 637-654. doi:10.1086/260062

[9] R. C. Merton, “Theory of Rational Option Pricing,” Journal of Economics and Management Science, Vol. 4, No. 1, 1973, pp. 141-183.

[10] D. Madan and E. Seneta, "The Variance Gamma(VG) Model for Share Market Returns," Journal of Business,
Vol. 63, No. 4, 1990, pp. 511-524. doi:10.1086/296519

[11] D. Madan and F. Milne, "Option Pricing with V.G. Martingale Components,” Mathematical Finance, Vol. 1, 1991, pp. 39-55. doi:10.1111/j.1467-9965.1991.tb00018.x

[12] D. Madan, P. Carr and E. Chang. "The Variance Gamma Processes and Option Pricing," European Finance Review, Vol. 2, No. 1, 1998, pp. 79-10. doi:10.1023/A:1009703431535

[13] M. C. Fu, "Variance-Gamma and Monte Carlo,” Advances in Mathematical Finance, Springer, 2007, pp. 21-35. doi:10.1007/978-0-8176-4545-8_2

[14] L. Cao and M. C. Fu, "Estimating Greeks for Variance Gamma," Proceedings of the 2010 Winter Simulation Conference, Baltimore, 5-8 December 2010, pp. 26202628. doi:10.1109/WSC. 2010.5678958

[15] L. Cao and Z. F. Guo, “Applying Gradient Estimation Technique to Estimate Gradients of European call following Variance-Gamma," Proceedings of Global Conference on Business and Finance, Nevada, 2-5 January 2011.

[16] L. Cao and Z. F. Guo, “A Comparison of Gradient Estimation Techniques for European Call Options,” Accounting \& Taxation, Forthcoming, 2011.

[17] L. Cao and Z. F. Guo, “A Comparison of Delta Hedging under Two Price Distribution Assumptions by Likelihood Ratio," International Journal of Business and Finance Research, Forthcoming, 2011.

[18] L. Cao and Z. F. Guo, "Delta Hedging with Deltas from a Geometric Brownian Motion Process," Proceedings of International Conference on Applied Financial Economic, Samos Island, March 2011. 